\title{
Efficacy of endoscopic ultrasound-guided fine-needle aspiration for esophageal schwannoma
}

\author{
Shigenaga Matsui ${ }^{a}$, Tomohiro Yamazaki ${ }^{a}$, Osamu Shiraishi ${ }^{b}$, Masatoshi Kudo ${ }^{a}$ \\ Kindai University Faculty of Medicine, Japan
}

A 50-year-old man was admitted to our hospital because of a protruding smooth mass in the mid-esophagus that had been detected on a screening barium esophagogram (Fig. 1A). Gastrointestinal endoscopy revealed a submucosal tumor (SMT) without ulceration in the distal third of the esophagus (Fig. 1B). Endoscopic ultrasonography (EUS) demonstrated a well-demarcated 20-mm hypoechoic tumor that originated from the muscularis propria layer (Fig. 1C). EUS-guided fine-needle $(22 \mathrm{G})$ aspiration (FNA) was subsequently performed (Fig. 1D).

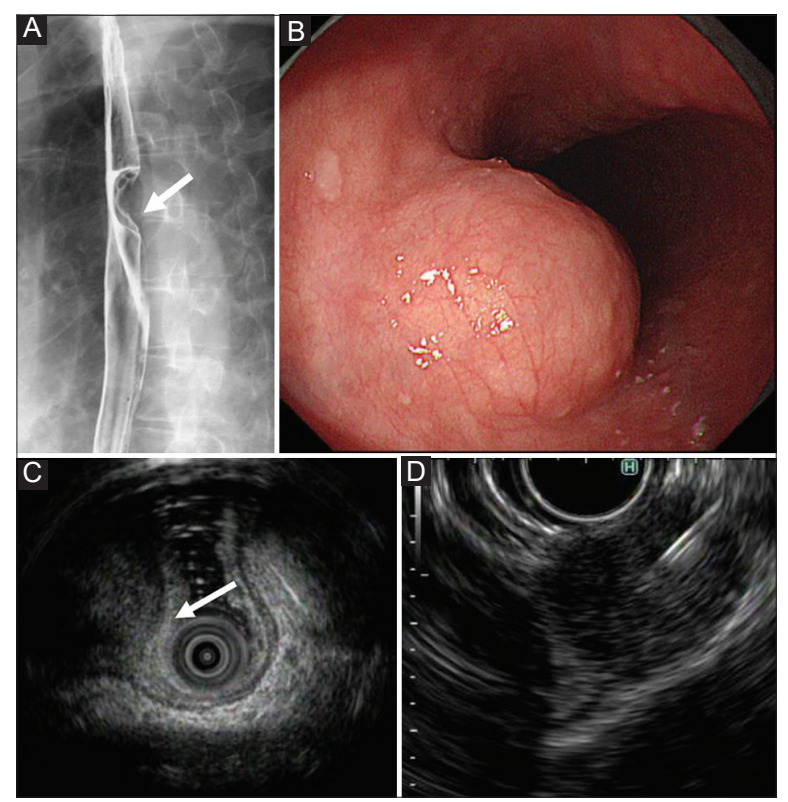

Figure 1 (A) Protruding smooth mass in the middle esophagus, detected on a screening barium esophagogram (arrow). (B) Gastrointestinal endoscopy revealed a submucosal tumor without ulceration. (C) Endoscopic ultrasonography demonstrated a well-demarcated 20-mm hypoechoic tumor, originating from the muscularis propria layer (arrow). (D) Endoscopic ultrasound-guided fine-needle (22 G) aspiration for the tumor

Department of a Gastroenterology and Hepatology (Shigenaga Matsui, Tomohiro Yamazaki, Masatoshi Kudo); bSurgery (Osamu Shiraishi), Kindai University Faculty of Medicine, Japan

\section{Conflict of Interest: None}

Correspondence to: Shigenaga Matsui, MD, PhD, Department of Gastroenterology and Hepatology, Kindai University Faculty of medicine, Osaka, Japan, e-mail: ma2i@med.kindai.ac.jp

Received 6 March 2021; accepted 28 March 2021; published online 3 June 2021

DOI: https://doi.org/10.20524/aog.2021.0636
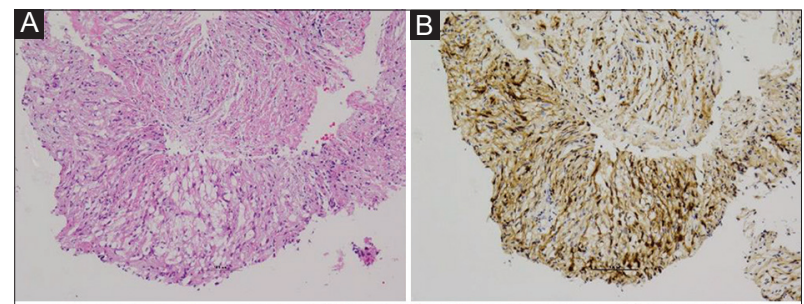

C

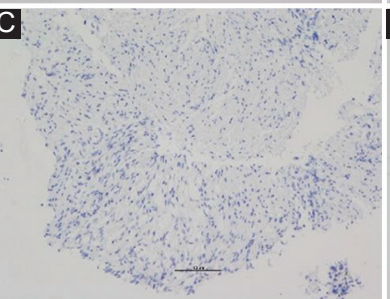

D

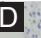

Figure 2 (A) Pathological examination (hematoxylin \& eosin staining $\times 100$ ) of the tumor specimen revealed spindle-shaped cells in a fasciculated pattern. Immunohistochemical staining revealed that the tumor cells were positive for S-100 proteins (B), but negative for desmin (C) and c-kit (D)

Pathological examination (hematoxylin \& eosin staining) of the tumor specimen revealed spindle-shaped cells in a fasciculated pattern (Fig. 2A). Immunohistochemical staining revealed that the tumor cells were positive for S-100 proteins, but negative for desmin and c-kit (Fig. 2B,C,D). The patient's diagnosis of esophageal schwannoma was confirmed. Thus, thoracoscopic surgery was performed to remove the tumor.

Esophageal schwannomas are rare esophageal submucosal tumors, comprising approximately $2 \%$ of esophageal tumors [1]. Esophageal schwannomas are difficult to diagnose definitively during preoperative endoscopy and imaging investigations, and the final diagnosis is confirmed by pathological examination of the surgically resected material. EUS-FNA is an effective and safe tissue sampling technique for gastrointestinal SMT diagnosis [2], and is useful as a diagnostic tool for esophageal schwannoma.

\section{References}

1. Shin S, Choi YS, Shim YM, Kim HK, Kim K, Kim J. Enucleation of esophageal submucosal tumors: a single institution's experience. Ann Thorac Surg 2014;97:454-459.

2. Rong L, Kida M, Yamauchi H, et al. Factors affecting the diagnostic accuracy of endoscopic ultrasonography-guided fine-needle aspiration (EUS-FNA) for upper gastrointestinal submucosal or extraluminal solid mass lesions. Dig Endosc 2012;24:358-363. 